\title{
Schuldentragfähigkeit in der Eurozone bei niedrigen bzw. negativen Zinsen
}

\author{
Wer soll das bezahlen? - eine Frage, die sich in Anbetracht der gegen die Corona-Krise \\ geschnürten Fiskalpakete derzeit viele stellen. Die Staatsausgaben für die Maßnahmenpakete \\ zur Stabilisierung der Wirtschaft sind enorm. Gleichzeitig sinken die Steuereinnahmen. \\ An Lösungsvorschlägen mangelt es nicht: von „Helikoptergeld“ der Zentralbanken \\ bis „Lastenausgleich“ per Vermögensteuern. Dabei hilft bereits das Niedrig- bzw. \\ Negativzinsumfeld, die weiter steigenden Schuldenberge abzutragen.
}

\begin{abstract}
Die fiskalischen Gegenmaßnahmen auf der Ebene der G20-Staaten haben ein beispielloses Volumen erreicht. Die US-Regierung hat Ausgabenpakete in Höhe von 8,3\% des Bruttoinlandsprodukts (BIP) verabschiedet. Dazu kommen Garantien und Kredite in Höhe von 2,4 \% des BIP. Die geplanten Fiskalpakete in der Europäischen Union belaufen sich auf knapp $21 \%$ des BIP. In Großbritannien sind es $27 \%$. Die Pakete unterscheiden sich stark, sowohl in ihrer Höhe als auch in ihrer Zusammensetzung. Dabei handelt es sich um unmittelbar ausgabenwirksame Maßnahmen, aber auch um Garantien und Kreditbürgschaften. Diese können bei Bedarf gezogen werden, um Schieflagen zu vermeiden. Sicher ist schon jetzt: Auch von Seiten der öffentlichen Schulden wird die Welt nach der Krise eine andere sein.
\end{abstract}

Es stellt sich die Frage nach der Schuldentragfähigkeit. Was dabei nicht übersehen werden sollte, ist die entlastende Wirkung extrem niedriger, sogar negativer Anleiherenditen. Wer sich zu günstigen Bedingungen refinan-

(c) Der/die Autor(en) 2020. Open Access: Dieser Artikel wird unter der Creative Commons Namensnennung 4.0 International Lizenz (https:// creativecommons.org/licenses/by/4.0/deed.de) veröffentlicht.

Open Access wird durch die ZBW - Leibniz-Informationszentrum Wirtschaft gefördert.

1 Zu den ökonomischen Auswirkungen der Corona-Pandemie vgl. Holtemöller et al. (2020) und Bofinger et al. (2020).

Dr. Hans-Jörg Naumer leitet die Abteilung Global Capital Markets \& Thematic Research bei der Investmentfondsgesellschaft Allianz Global Investors in Frankfurt am Main. zieren kann, braucht das Abtragen des Schuldenbergs wenig zu fürchten. Im Idealfall trägt sich dieser - Renditen, Inflation und Wachstum sei Dank - über die Zeit selbst ab. Tatsächlich ist der „Club der negativen Zinsen“ kontinuierlich weitergewachsen (vgl. Abbildung 1). Bei deutschen Staatsanleihen gibt es über fast alle Laufzeiten hinweg keine positive Rendite mehr. Auch in anderen europäischen wie außereuropäischen Staaten gehören Negativrenditen zum bekannten Bild an den Anleihemärkten. Im globalen Kontext notieren Ende Mai 2020 knapp $60 \%$ des globalen Staatsanleihenmarktes mit einer Rendite von unter 1\%. Knapp $20 \%$ aller Staatsanleihen haben eine negative Rendite - weltweit, nominal. Der Kaufkraftverlust durch Inflation, und sei diese noch so niedrig, wurde dabei noch gar nicht berücksichtigt.

Wirkung der niedrigen bzw. negativen Zinsen

Wie komfortabel niedrige bzw. negative Zinsen sein können, zeigt sich bei den impliziten Zinsen, welche die Staaten auf ihre Schulden zahlen müssen. ${ }^{2}$ Vergleicht man den Durchschnitt der impliziten Zinsen, die seit dem Ausbruch der Finanzkrise bis an den aktuellen Rand (2008 bis 2020) anfielen, mit dem Durchschnitt des 10-Jahreszeitraums davor (1997 bis 2007), so zeigt sich für Deutschland, Frankreich und Italien ein (nahezu) halbierter Zins (vgl. Tabelle 1). Auch für Spanien ist der implizite Zins deutlich zurückgegangen. Verglichen mit dem von der Europäischen Kommission für das Jahr 2020 angenommenen impliziten Zins liegen diese noch einmal deutlich darunter.

Kann dieses Renditeniveau dazu beitragen, dass die Staaten aus ihren Schulden über die Zeit herauswachsen? In einer Szenariobetrachtung wird dies im Folgenden

2 Unter „impliziten Zinsen“ werden hier die Zinsen verstanden, die von der Regierung im Durchschnitt auf alle ausstehenden Anleihen für den Schuldendienst gezahlt werden müssen. 


\section{Abbildung 1}

Renditeanteil an der Marktkapitalisierung bei

Anleihen

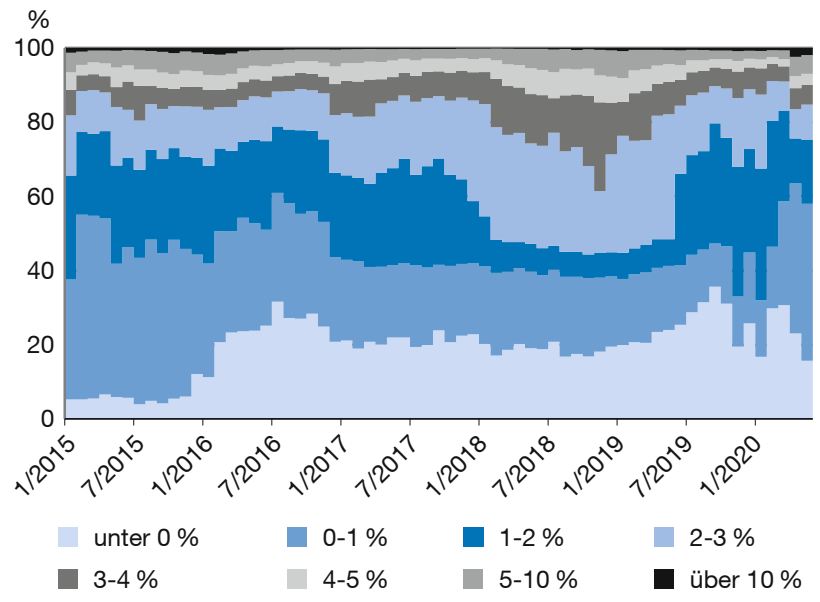

Die Wertentwicklung der Vergangenheit ist kein verlässlicher Indikator für die zukünftige Wertentwicklung.

Quellen: Bloomberg; ICE BofAML Global Fixed Income Markets Index; AllianzGI Global Capital Markets, Stand: 26. Mai 2020.

für vier Länder der Eurozone (Deutschland, Frankreich, Spanien und Italien) untersucht. Die Grundüberlegungen bauen auf Contessi (2012) auf, der wiederum auf Domar (1944) zurückgreift. Zentral ist das Verhältnis von dem realen Zins, mit dem die öffentlichen Schulden refinanziert werden, zu der realen volkswirtschaftlichen Wachstumsrate. Wird ein ausgeglichener Primärhaushalt unterstellt, ist für die Schuldendynamik entscheidend, dass der Realzins langfristig unterhalb der realen Wachstumsrate liegt. Nur dann kommt es zu einem Abbau der Staatsschulden über die Zeit. Die öffentliche Hand wächst gleichsam aus ihren Schulden heraus, d.h., die Schuldenquote sinkt. Ist dies nicht der Fall, müssen Primärüberschüsse erzielt werden. Um den Schuldenabbau voranzubringen, muss dieser umso größer sein, je stärker der Realzins über dem Wachstum liegt. ${ }^{3}$

\section{Mechanik des Schuldenabbaus}

Für die Szenariobetrachtungen wurde die Entwicklung der Schuldenquoten entlang der Formel

$d_{t+1}=\left(1+r_{t+1}\right) /\left(1+g_{t+1}\right) * d_{t}-p b_{t+1}$

mit $r_{t+1}=n_{t+1}-p_{t+1}, d=$ Schuldenquote, $n=$ nominaler Zinssatz des Staates, $r=$ realer Zinssatz des Staates, $\mathrm{g}=$ reale Wachstumsrate, $\mathrm{pb}=$ Primärbilanz in Relation zum BIP und $p=$ Inflationsrate gemäß Contessi und Do-

3 Vgl. dazu auch eine vorausgehende Studie von Naumer (2015).
Tabelle 1

Durchschnittliche implizite Zinsen

in \%

\begin{tabular}{lcccc} 
& 2020 & $\begin{array}{c}2008 \text { bis } \\
2020\end{array}$ & $\begin{array}{c}1997 \text { bis } \\
2007\end{array}$ & $\begin{array}{c}\text { Rückgang über } \\
\text { die Vergleichs- } \\
\text { perioden }\end{array}$ \\
\hline Deutschland & 1,36 & 2,44 & 5,02 & 51,41 \\
\hline Frankreich & 1,36 & 2,55 & 4,97 & 48,64 \\
\hline Spanien & 2,25 & 3,38 & 5,30 & 27,78 \\
\hline Italien & 2,44 & 3,44 & 5,54 & 52,43 \\
\hline
\end{tabular}

${ }^{1}$ Rückgang des durchschnittlichen impliziten Zinses in \% im Vergleich 1997 bis 2007 zu 2008 bis 2020.

Quellen: Europäische Kommission; AllianzGI Global Capital Markets \& Thematic Research; Stand: Mai 2020.

mar untersucht. ${ }^{4}$ Die Schuldenquote „d“ setzt den Schuldenstand des betrachteten Landes in Relation zum BIP. Ausgangspunkt für den aktuellen Zeitrand der Jahre 2020 und 2021 waren die Schätzungen für die Schuldenquoten der Europäischen Kommission vom Mai 2020 (also inklusive der im Kontext der Pandemie bereits beschlossenen fiskalisch wirksamen Maßnahmen). ${ }^{5}$ Davon ausgehend wurden entlang dieser Formel die Auswirkungen (1) der realen impliziten Zinsen „r", mittels derer sich die öffentlichen Haushalte refinanzieren, (2) der Primärbilanz „pb“ (in Relation zum Wachstum), die über die Betrachtungszeiträume zunächst als ausgeglichen unterstellt wurde, und (3) des (realen) Wachstums "g" analysiert. Für die weitere Betrachtung der einzelnen Varianten wurden Annahmen bezüglich der Inflation „p“ und des Primärhaushalts „pb“ getroffen. Als Annahme für das reale Wachstum wurden die langfristigen Schätzungen der OECD verwendet, die bis zum Jahr 2060 reichen. ${ }^{6}$ Die Modellierung der impliziten Zinssätze, die auf die jeweiligen Haushalte entfallen, setzt an den aktuellen Schätzungen für das Jahr 2021 der Europäischen Kommission an (vgl. Tabelle 1).

4 Anders als bei Contessi wurde der Münzgewinn der Zentralbank in der Gleichung nicht gesondert betrachtet, sondern implizit in den Primärhaushalt einberechnet.

5 Dabei wurden nur die direkt fiskalisch wirksamen Ausgaben infolge der Corona-Krise in die Betrachtungen einbezogen, nicht möglicherweise haushälterisch noch wirksam werdende Maßnahmen, wie z.B. Bürgschaften und Kredite. Hiervon können weitere Belastungen ausgehen, welche die Schuldenrelationen verschlechtern. Auch wurden die expliziten Staatsschulden als Ausgangspunkt genommen, nicht die erst in Zukunft wirksam werdenden impliziten Ausgaben, die sich z. B. aus den Sozialversicherungen ergeben. Die Kommission betont dabei, dass sie lediglich von einer Infektionswelle ausgeht. Würde eine weitere folgen, triebe dies die Schulden entsprechend weiter in die Höhe. Zur Veranschaulichung wurden zusätzlich die historischen Schuldenquoten gemäß der „Historical Public Debt Database“ des Internationalen Währungsfonds (IWF) genutzt.

6 OECD Daten, „GDP long-term forecast“, Stand: 25. Mai 2020. 


\section{Abbildung 2}

Entwicklung der Schuldenquote in Deutschland und Italien bei ausgeglichenem Primärhaushalt
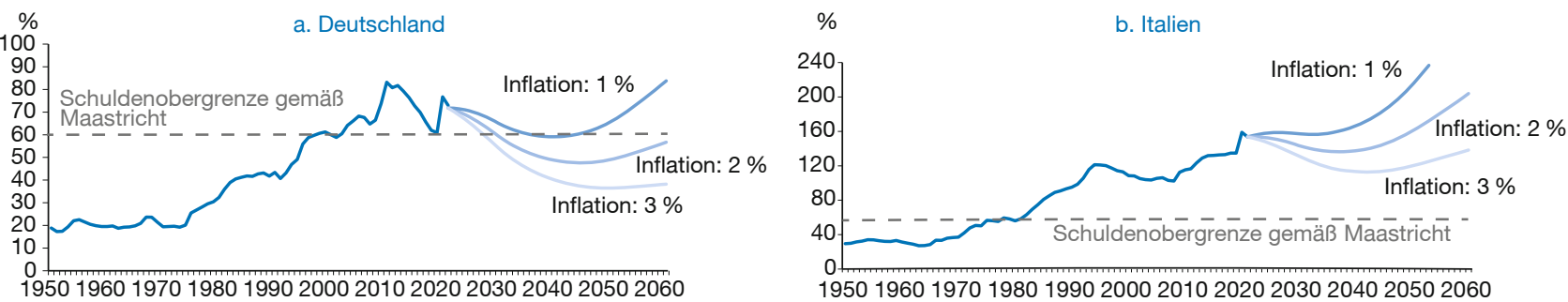

Quellen: IMF (International Monetary Fund) Historical Public Debt Database; Europäische Kommission Mai 2020; AllianzGI Global Capital Markets \& Thematic Research.

Bei den Annahmen zu den zukünftigen Veränderungen des impliziten Zinssatzes auf die Staatsverschuldung wurde modellhaft berücksichtigt, dass dieser mit einem nachlaufenden Effekt auf Veränderungen der Zinsstrukturkurve reagiert. Das heißt: Renditeveränderungen an den Kapitalmärkten kommen nur zeitverzögert mittels Refinanzierung auslaufender Staatsanleihen bei den Finanzierungskosten für die öffentlichen Schulden an.

Für die jeweiligen Länder wurde deshalb zunächst beim impliziten Zins ein gleitender Rückgang p.a. bis zu einem Tief im Jahr 2030 unterstellt, der dem durchschnittlichen Rückgang im jeweiligen Land in den Jahren 2008 bis 2020 entspricht. Dieser Rückgang lag, von Land zu Land variierend, von leicht über 20 bis leicht unter 30 Basispunkten. Der ab 2031 dann gemäß Modell einsetzende Anstieg des impliziten Zinses vollzieht sich dann in der Simulation bis zum Jahr 2060. Als Annäherung für ein „normales“ Zinsumfeld wurde die „Ultimate Forward Rate“ der European Insurance and Occupational Pensions Authority (EIOPA, 2014, 2020) gewählt, wie sie gemäß der Regularien für Solvency II zum Einsatz kommt. Modellhaft wurde unterstellt, dass die impliziten Zinsen ab 2030 um 20 Basispunkte p.a. wieder ansteigen und dann eine Kappungsgrenze bei diesem langfristigen Zins gemäß EIOPA haben.

Da die Bundesanleihen de facto eine Benchmark für die Bepreisung der Anleihen aus den anderen Ländern des Euroraums darstellen, wurden für diese Renditezuschläge auf den langfristigen Zins einberechnet, die dem durchschnittlichen Renditezuschlag p. a. des jeweiligen Landes im Vergleich zu Deutschland der Jahre 2008 bis 2020 entsprechen. Damit sollte auch den Abweichungen bei der durchschnittlichen Duration der den Haushalten unterliegenden Staatsanleihen Rechnung getragen werden. Es wurde unterstellt, dass es über die nächsten Jahre/ Jahrzehnte zu keinen Bonitätsveränderungen kommt, also auch zu keinen Verschlechterungen, die zu steigenden Bonitätszuschlägen und damit steigenden Zinsen führen würde.

\section{Szenario 1: ausgeglichener Primärhaushalt}

Die entlastende Wirkung weiter sinkender impliziter Zinsen wird bei jedem der betrachteten Länder sichtbar - wenn auch sehr unterschiedlich. Sie ist abhängig von der Höhe der Schuldenquote zu Beginn der Betrachtung. Dies zeigt das Basisszenario, bei dem ein ausgeglichener Primärhaushalt unterstellt wird. Dabei gilt: Je höher der anfängliche Schuldenstand, desto weniger stark ausgeprägt ist die Wirkung. Und je höher die durchschnittliche Inflationsrate, desto besser gelingt der Schuldenabbau. Bei der Inflation wurde ein Korridor von $+/-1 \%$ um das Inflationsziel der Europäischen Zentralbank untersucht.

In Deutschland sinkt die Schuldenquote bei einer unterstellten Inflationsrate von $3 \%$ kontinuierlich (vgl. Abbildung 2a). Bei durchschnittlichen $2 \%$ sinkt sie bis 2044 auf leicht unter $48 \%$ und steigt dann langsam wieder an. Bei einer Inflationsrate von durchschnittlich $1 \%$ wird das Maastricht-Kriterium von $60 \%$ im Jahr 2036 unterschritten. Danach sinkt die Schuldenquote bis 2041 leicht weiter, um erst dann wieder anzusteigen. Auch in Frankreich sinken die Schuldenquoten in Abhängigkeit von der unterstellten Inflationsrate zunächst sehr deutlich. Zu einer dauerhaften Stabilisierung kommt es aber nur bei $3 \%$ Inflation. Bei einer Inflationsrate von $2 \%$ sinkt die Schuldenquote bis 2046 auf unter $70 \%$. Bei einer Inflationsrate von $1 \%$ fällt sie auf ein Tief von knapp unter $88 \%$ (2042), um dann bis zum Ende der Betrachtungsperiode auf $120 \%$ anzusteigen.

In Spanien kommt es in allen drei Inflationsszenarien nur temporär zu Rückgängen der Schuldenquoten. Allerdings sind diese Rückgänge deutlich schwächer ausgeprägt als z. B. im Fall von Deutschland oder Frankreich. Die niedrigste Schuldenquote wird bei unterstellten $3 \%$ Inflation im Jahr 2043 mit leicht unter $81 \%$ erreicht. Werden nur $2 \%$ Inflation unterstellt, können bestenfalls $98 \%$ (2038) knapp geschafft werden. Im Fall Italiens bedarf es einer ausgeprägten Inflationsrate von $3 \%$, um einen merklichen Rückgang der Schuldenquote zu verzeichnen (vgl. Abbildung 2b). 2042 wird ein 
Abbildung 3

Entwicklung der Schuldenquote in Deutschland und Italien bei einem Primärüberschuss von $1 \%$ p.a.
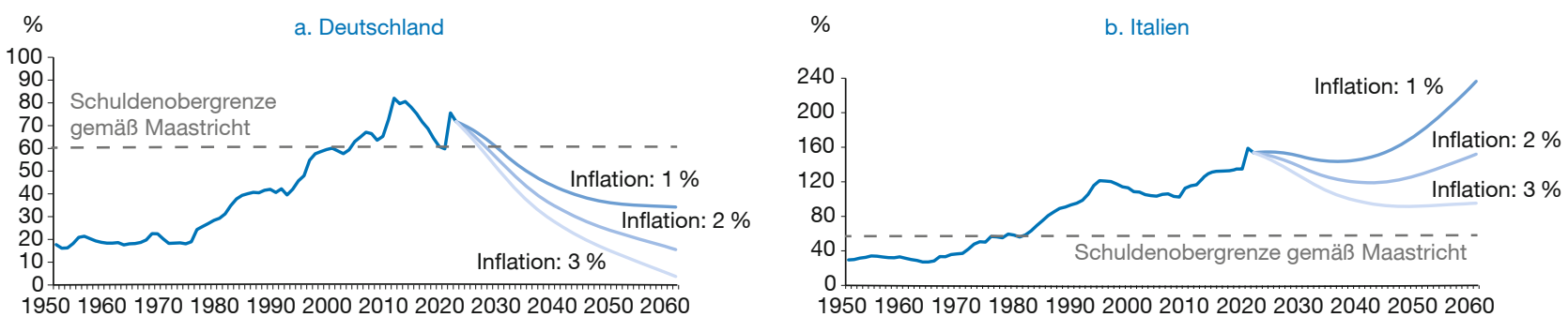

Quellen: IMF (International Monetary Fund) Historical Public Debt Database; Europäische Kommission Mai 2020; AllianzGI Global Capital Markets \& Thematic Research.

zwischenzeitlicher Tiefpunkt von $113 \%$ erreicht, bevor dann der Wiederanstieg beginnt. Bei einer nur $2 \%$ igen Inflationsrate wird die Schuldenquote ihren tiefsten Punkt im Jahr 2037 mit leicht über $133 \%$ erreichen. Danach steigt diese auf 204 (2060) an. Bei der geringsten Inflationsrate von 1\% wächst die Schuldenquote kontinuierlich auf $300 \%$ im Jahr 2060 an. Es bedarf also deutlich weiterer Anstrengungen, um zu einer Schuldenreduktion zu gelangen.

\section{Szenario 2: positiver Primärhaushalt}

Ein deutlicher Kurswechsel bei der Schuldendynamik kann mit einem dauerhaften Primärüberschuss von $1 \%$ pro Jahr erzielt werden. Im Fall Deutschlands kommt es zu kontinuierlichen Rückgängen der Schuldenquote für jedes der drei Inflationsszenarien (vgl. Abbildung 3a). Bei $3 \%$ Inflation sind die Schulden bis 2060 faktisch abgebaut. Bei einer nur $1 \%$ igen Inflationsrate sinkt die Schuldenquote bis zum Ende des Betrachtungszeitraums auf rund $34 \%$. Frankreich kann seine Schuldenquote bei $3 \%$ Inflation auf rund $21 \%$ (2060) reduzieren. Bei einer Inflationsrate von nur $1 \%$ wird der tiefste Punkt der Schuldenquote mit knapp unter $66 \%$ im Jahr 2049 erreicht. Danach beginnt der Wiederanstieg auf $73 \%$ (2060). Spanien kann ebenfalls Rückgänge bei der Schuldenquote erreichen, wobei diese nur bei einer Inflationsrate von 3\% dauerhaft sind. Bei einer Inflation von lediglich $1 \%$ wird ein Rückgang auf $99 \%$ im Jahr 2038 erreicht. Danach setzt ein Wiederanstieg auf $150 \%$ ein. Bei Italien ergibt sich für keines der Inflationsszenarien ein dauerhafter Abbau der Schuldenquote, trotz jährlicher Primärüberschüsse (vgl. Abbildung 3b). Bei einer Inflation von jahresdurchschnittlich $1 \%$ wird zunächst ein Rückgang auf $144 \%$ im Jahr 2034 erreicht. Danach setzt ein Wiederanstieg auf $236 \%$ bis 2060 ein.

Szenario 3: ausgeglichener Primärhaushalt, länger andauernder Rückgang der impliziten Zinsen

Die Bedeutung niedriger impliziter Zinsen wird noch deutlicher, wenn unterstellt wird, der Zinsrückgang würde fünf
Jahre länger, also bis einschließlich 2035 anhalten, bevor die Zinsen dann langsam wieder ansteigen. Um die Darstellung abzukürzen, beschränkt sich die Betrachtung auf Deutschland und Italien, die beiden Länder mit der jeweils niedrigsten bzw. höchsten Schuldenquote der hier betrachteten Ländergruppe. Auch ohne einen zusätzlichen Primärüberschuss gelingt Deutschland in diesem Fall eine merkliche Senkung der Schuldenquote (vgl. Abbildung 4a). 2060 steht sie bei einer Inflationsrate von $2 \%$ bei $32 \%$. Beläuft sich die Inflation nur auf $1 \%$, liegt sie bei $48 \%$. Am Beispiel Italiens kommt es bei $1 \%$ Inflation zu einem Rückgang auf $141 \%$ im Jahr 2042, bevor ein dynamischer Wiederanstieg beginnt (vgl. Abbildung 4b). Liegt die Inflation bei $2 \%$, gelingt ein Rückgang auf $111 \%$ (2047). Dann setzt ein Anstieg auf $131 \%$ bis 2060 ein.

\section{Schuldenabbau per Niedrigzinsen}

Entscheidend beim Schuldenabbau ist die entlastende Wirkung niedriger impliziter Zinsen. Diese wird für jedes der betrachteten Länder spürbar. Allerdings kommt es im Fall Italiens ohne zusätzlichen Primärüberschuss nur bei einer Inflationsrate, die dauerhaft über der Zielgröße der EZB liegt, zu einer rückläufigen Schuldenquote. Die weitere Entwicklung hängt dabei sehr deutlich von der zukünftigen Höhe der impliziten Zinsen ab.

Zur Verdeutlichung noch einmal folgende Betrachtung am Beispiel Deutschlands, dem Land mit dem günstigsten Ausgangspunkt was Schuldenquote und Schuldzins betrifft. Läge der implizite Zins für das Jahr 2020 statt bei 1,36\% (Europäische Kommission) bei 2,44\% (was dem Durchschnitt des impliziten Zinses der Jahre 2008 bis 2020 entspricht), verliefe der Rückgang der Schuldenquote schon merklich ungünstiger. Ohne weitere Maßnahmen würde bei einer Inflation von $1 \%$ eine Schuldenquote von $100 \%$ im Jahr 2053 erreicht. Läge der implizite Zins gar bei - unwahrscheinlichen - 5\% (was dem impliziten Zins der Jahre 1997 bis 2007 entspricht), würden die Schuldenquoten unabhängig von der Inflationsentwicklung 


\section{Abbildung 4}

Entwicklung der Schuldenquote in Deutschland und Italien bei ausgeglichenem Primärhaushalt und einem Rückgang der impliziten Zinsen bis 2035
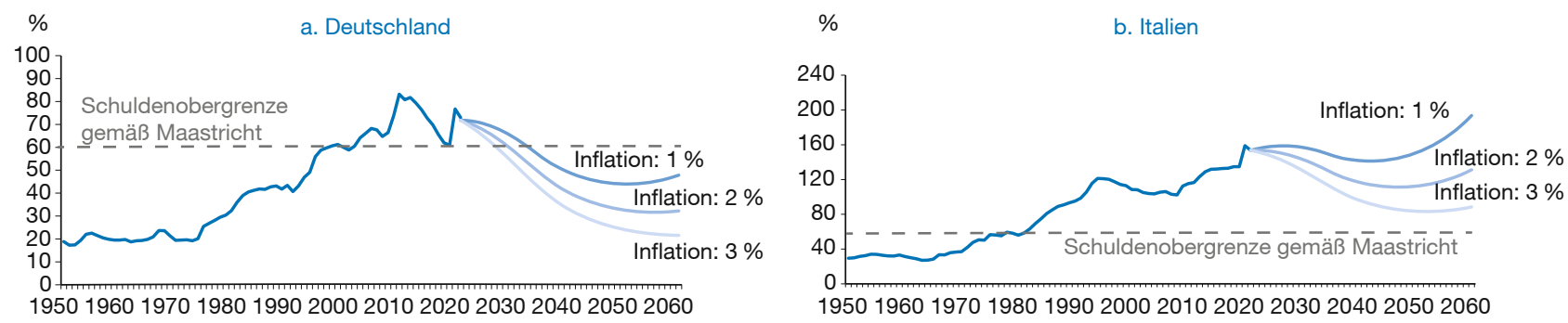

Quellen: IMF (International Monetary Fund) Historical Public Debt Database; Europäische Kommission Mai 2020; AllianzGI Global Capital Markets \& Thematic Research.

kontinuierlich ansteigen. Ein Primärüberschuss von $1 \%$ würde nur bei einer Inflationsrate von $3 \%$ zu einem dauerhaften Rückgang der Schuldenquote führen. Auch eine mittlere Variante von 3,75\% würde den Schuldenabbau merklich hemmen. In diesem Fall gelänge ein anhaltender Rückgang der Schuldenquote nur in dem unwahrscheinlichen Fall einer Inflationsrate, die dauerhaft bei $3 \%$ p.a. liegt, und das von 2021 an.

Eine Entschuldung gelingt umso besser, je höher die Inflation und je niedriger die Nominalrenditen - bzw. je länger die Phase niedriger bzw. negativer Zinsen anhält. Am Ende bezahlen die Gläubiger, d.h. die Zeichner von Staatsanleihen, für einen Teil des Schuldenabbaus selbst: indem sie zu Realzinsen anlegen, die unterhalb des realen Wachstums liegen.

\section{Deutschland und das neue Konjunkturpaket}

Deutschland schafft - hauptsächlich dank extrem niedriger bzw. negativer Zinsen - den Abstieg vom Schuldengipfel. Zusätzliche Steuern in Verbindung mit einem günstigen Ausgangsniveau bei der Schuldenquote sind nicht notwendig, auch nicht, wenn das Anfang Juni beschlossene Konjunkturpaket von 130 Mrd. Euro mit berücksichtigt wird, was in den hier vorgelegten Betrachtungen noch nicht einbezogen wurde. Die 130 Mrd. Euro entsprechen knapp $4 \%$ des deutschen BIP. Würden diese - stark vereinfachend - verteilt über die Jahre 2020 und 2021 hälftig ausgegeben, stiege die Schuldenquote in diesen beiden Jahren um jeweils knapp 2 Prozentpunkte - und hätte damit kaum einen Effekt auf den Verlauf der Schuldenpfade.

\section{Literatur}

Bofinger, P., S. Dullien, G. Felbermayr, C. Fuest, M. Hüther, J. Südekum und B. Weder di Mauro (2020), Wirtschaftliche Implikationen der Corona-Krise und wirtschaftspolitische Maßnahmen, Wirtschaftsdienst, 100(4), 259-265, https://www.wirtschaftsdienst.eu/inhalt/jahr/2020/ heft/4/beitrag/wirtschaftliche-implikationen-der-corona-krise-undwirtschaftspolitische-massnahmen.html (23. Juli 2020).

Contessi, S. (2012), An Application of Conventional Sovereign Debt Sustainability Analysis to the Current Debt Crises, Review, 94(3), https:// doi.org/10.20955/r.94.197-220 (23. Juli 2020).

Domar, E. D. (1944), The „Burden of the Debt" and the National Income, The American Economic Review, 34(4), 798-827m, http://www.jstor. org/stable/1807397 (23. Juli 2020).

EIOPA (2014), CP-14-042 Consultation paper on a Technical document regarding the risk free interest rate term structure, EIOPA, https:// register.eiopa.europa.eu/Pages/Consultations/CP-14-042-Consultation-paper-on-a-Technical-document-regarding-the-risk-free-interest-rate-term-structure.aspx (23. Juli 2020).

EIOPA (2020), Risk-free interest rate term structures | Eiopa, EIOPA, https://www.eiopa.europa.eu/tools-and-data/risk-free-interest-rateterm-structures_en (23. Juli 2020).

Holtemöller, O., S. Kooths, C. Michelsen, T. Schmidt und T. Wollmershäuser (2020), Wirtschaft unter Schock - Finanzpolitik hält dagegen, Wirtschaftsdienst, 100(4), 254-258, https://www.wirtschaftsdienst.eu/ inhalt/jahr/2020/heft/4/beitrag/gemeinschaftsdiagnose-wirtschaftunter-schock-finanzpolitik-haelt-dagegen.html (23. Juli 2020).

Naumer, H.-J. (2015), Fördern Geldpolitik und Niedrigzinsen einen nachhaltigen Rückgang der Staatsschulden?, Wirtschaftsdienst, 95(9), 614-618, https://www.wirtschaftsdienst.eu/inhalt/jahr/2015/heft/9/ beitrag/foerdern-geldpolitik-und-niedrigzinsen-einen-nachhaltigenrueckgang-der-staatsschulden.html (23. Juli 2020).

Title: Debt Sustainability in the Euro Area When Interest Rates Are Low or Negative

Abstract: Who is going to pay? That question is heard regularly in connection with corona-related fiscal packages. Quite rightly. Governments have adopted huge packages to stabilise their economies. While there is no shortage of suggestions of how the new public expenditure and old debt is to be funded, many people seem to be overlooking the fact that the current low or negative interest rate environment is already making a major contribution to debt reduction.

JEL Classification: E50, G10, H62 